\title{
$\beta$-decay data requirements for reactor decay heat calculations: study of the possible source of the gamma-ray discrepancy in reactor heat summation calculations
}

\author{
A. Sonzogni ${ }^{9}$, and K. Peräjärvi ${ }^{10}$ \\ 1 IFIC-Univ. Valencia, Valencia, Spain \\ 2 Institute of Nuclear Research, Debrecen, Hungary \\ 3 University of Jyväskylä, Jyväskylä, Finland \\ 4 GSI, Darmstadt, Germany \\ 5 PNPI, Gatchina, Russia \\ ${ }^{6}$ University of Surrey, Guildford, UK \\ ${ }^{7}$ Mushashi Institute of Technology, Japan \\ 8 IAEA Nuclear Data Section, Vienna, Austria \\ 9 BNL, USA \\ 10 STUK, Helsinki, Finland
}

A. Algora ${ }^{1,2}$, J.L. Taín ${ }^{1}$, A.B. Perez-Cerdan ${ }^{1}$, B. Rubio ${ }^{1}$, J. Agramunt ${ }^{1}$, L. Caballero ${ }^{1}$, E. Nácher ${ }^{1}$, D. Jordan ${ }^{1}$, F. Molina ${ }^{1}$, A. Krasznahorkay ${ }^{2}$, M.D. Hunyadi ${ }^{2}$, J. Gulyás ${ }^{2}$, A. Vitéz ${ }^{2}$, M. Csatlós ${ }^{2}$, L. Csige ${ }^{2}$,

J. Äysto ${ }^{3}$, H. Penttilä ${ }^{3}$, S. Rinta-Antila ${ }^{3}$, I. Moore ${ }^{3}$, T. Eronen ${ }^{3}$, A. Jokinen ${ }^{3}$, A. Nieminen ${ }^{3}$, J. Hakala ${ }^{3}$, P. Karvonen ${ }^{3}$,

A. Kankainen ${ }^{3}$, U. Hager ${ }^{3}$, T. Sonoda ${ }^{3}$, A. Saastamoinen ${ }^{3}$, J. Rissanen ${ }^{3}$, T. Kessler ${ }^{3}$, C. Weber ${ }^{3}$, J. Ronkainen $^{3}$,

S. Rahaman ${ }^{3}$, V. Elomaa ${ }^{3}$, K. Burkard ${ }^{4}$, W. Hüller ${ }^{4}$, L. Batist ${ }^{5}$, W. Gelletly ${ }^{6}$, T. Yoshida ${ }^{7}$, A.L. Nichols ${ }^{8}$,

\begin{abstract}
The decay heat of fission products plays an important role in predictions of the heat up of nuclear fuel in reactors. The released energy is calculated as the summation of the activities of all fission products $P(t)=\sum E_{i} \lambda_{i} N_{i}(t)$, where $E_{i}$ is the decay energy of nuclide $i$ (gamma and beta component), $\lambda_{i}$ is the decay constant of nuclide $i$ and $N_{i}(t)$ is the number of nuclide $i$ at cooling time $t$. Even though the reproduction of the measured decay heat has improved in recent years, there is still a long standing discrepancy in the $t \sim 1000 \mathrm{~s}$ cooling time for some fuels. A possible explanation to this improper description has been found in the work of Yoshida et al. [1], where it has been shown that the incomplete knowledge of the $\beta$-decay of some Tc isotopes can be the source of the systematic discrepancy. Motivated by [1], we have recently measured the $\beta$-decay process of some Tc isotopes using a total absorption spectrometer at the IGISOL facility in Jyväskylä. The results of the measurements as well as the their consequences on summation calculations are discussed.
\end{abstract}

\section{Introduction}

Safe operational procedures are a major pre-requisite of the design and development of nuclear power plants. The primary aim of this work was to study the decay properties of specific nuclei that are important contributors to the so-called reactor decay heat emitted by the reactor core immediately after shutdown.

In a working nuclear reactor, approximately $8 \%$ of the total energy generated during the fission process is related to gamma and beta energy released in the natural decay of fission products, and it is commonly called decay heat [2]. Once the reactor is shutdown, the energy released in radioactive decay provides the main source of heating. Hence, coolant needs to be maintained after termination of the fission process in a reactor, and the form and extent of this essential requirement needs to be specified on the basis of what are called decay-heat calculations. Decay heat varies as a function of cooling time, and can be determined in theory from known nuclear data, with computations based on the inventory of nuclei created during the fission process and after reactor shutdown, and their radioactive decay characteristics. These calculations require large libraries of cross-sections, fission-yield and properly measured decay-data.

Most of the data used in nuclear decay databases suffer from the so called Pandemonium effect. This effect, which was introduced in the work of Hardy and coworkers [3], is related to the difficulties we face when we build a level scheme from a beta decay experiment using conventional (high resolution) techniques. The problem is related to the low efficiency of Ge detectors and to the difficulty to detect all the individual gamma rays that follow the beta decay. In a high resolution experiment, the beta probability to a certain level is determined from the intensity balance of the gamma-feeding and deexitation of the level. If there is a large fragmentation in the gamma intensity due to the high level density or if in the decay there are gamma rays of high energy involved, we can miss the detection of gamma rays. As a consequence, feeding at high excitation is not observed and incorrectly assigned to low lying levels. This effect has major consequences for decay heat calculations. Due to the effects of missing levels at high excitation energy there is an underestimate of the total $\gamma$ energy and an overestimate of the total $\beta$ energy released in the decay. At this point it is worth mentioning that the only 
way to avoid this problem is the application of total absorption techniques to $\beta$-decay studies. We have performed several experiments at GSI and ISOLDE using this technique [4], and new methods of analysis of the resulting data have been developed recently $[5,6]$.

Improvements in the world major data libraries for summation calculations have resulted in fairly good reproducibility of the integral-type measurements for different fissioning nuclei from ${ }^{233} \mathrm{U}$ to ${ }^{241} \mathrm{Pu}$. However, there remains a substantial discrepancy between the calculations and the experiments in the $\gamma$-ray component of the decay in the cooling range from 300 to $3000 \mathrm{~s}$ after an instantaneous fission event [1]. This effect was called by the authors [1] the $\gamma$-ray discrepancy.

It was shown in [1] that the $\gamma$-ray discrepancy occurs not just for the decay heat calculations for ${ }^{239} \mathrm{Pu}$, but also for $233,235,238 \mathrm{U}$ as well. This is the reason why the identification of the nuclei responsible for the discrepancy was considered a very important task by the authors. A careful study showed that the best candidates are ${ }^{102,104,105} \mathrm{Tc}$. The choice is justified by the following: a) they have a sizable amount of fission yield, b) their fission yields in the different fissionable nuclei are correlated as required, c) their half-lives satisfy the time condition given above and d) the $\beta$-decay $Q$-value is large enough to allow additional $\beta$-feeding in the high excitation region of the decay scheme of the daughter nuclei. To test the validity of the hypothesis a sizable amount of $\beta$-strength was artificially added at excitation energy $>4.5 \mathrm{MeV}$ for ${ }^{104} \mathrm{Tc}$ and $>2.5 \mathrm{MeV}$ for ${ }^{105} \mathrm{Tc}$ in [1]. As a result, the values of $E_{\gamma}^{i}$ increase by $0.3 \mathrm{MeV}$ and $0.7 \mathrm{MeV}$ for ${ }^{104} \mathrm{Tc}$ and ${ }^{105} \mathrm{Tc}$. The total effective increase solves two-thirds of the $\gamma$-ray discrepancy. It was also shown by Yoshida et al. that the strength required is fairly comparable to what is predicted by Gross Theory [7,8]. However we can only decide on the basis of experiment. This is why it was proposed to measure the $\beta$-decay of ${ }^{102,104,105} \mathrm{Tc}$ to $\mathrm{Ru}$ with the application of the total absorption technique.

\section{The experiment}

The Tc isotopes of interest are refractory elements which are difficult to extract from conventional ion sources. For that reason our experiments were performed at the Ion-Guide Isotope Separator On-Line (IGISOL) facility of the University of Jyväskylä. In the ion guide method, the nuclear reaction products recoiling out of a target are stopped in a gas (usually helium) and are transported by a gas flow through a differential pumping system directly into the acceleration stage of a mass separator. This process can be made fast enough for some reaction products to survive as singly-charged ions. Applying this technique, no ion source in the classical sense is used. The system is chemically insensitive which allows the extraction of refractory elements.

We have performed two experiments in this facility aimed to study the beta decay of isotopes related to the decay heat problem. In the first experiment we measured the beta decay of ${ }^{104,105} \mathrm{Tc}$ isotopes. In the second we have combined for the first time a total absorption spectrometer with the Penning trap available at IGISOL (JYFLTRAP) and measured the beta decay of ${ }^{101} \mathrm{Nb},{ }^{104,105,106,107} \mathrm{Tc}$ and ${ }^{101,102,105,106} \mathrm{Mo}$. In this last run in order to test the purity of our sources in the first experiment we repeated the measurement of the ${ }^{104,105} \mathrm{Tc}$ isotopes. The Penning trap is an excellent tool in this kind of experiments as a isotope separator (very high resolution mass separator) where the purity of the sources is of great importance.

In this work we will present the results of preliminary analysis of our first experiment. In this case a proton beam of $30 \mathrm{MeV}$ delivered by the Jyväskylä University Cyclotron was used to produce fission in a natural $U$ target of $15 \mathrm{mg} / \mathrm{cm}^{2}$. Typical currents were about $4 \mu \mathrm{A}$. The nuclei of interest were separated using the IGISOL facility. The separated activity was carried to the Total Absorption Gamma Spectrometer (TAGS) by the tape transport system. The Tags used was designed at the Nuclear Institute of St. Petersburg. It consist of two NaI(Tl) cylindrical crystals. The larger crystal has dimension: $\phi=200 \mathrm{~mm} \times l=200 \mathrm{~mm}$, and it has a longitudinal hole of $\phi=43 \mathrm{~mm}$. The other crystal has dimensions: $\phi=200 \mathrm{~mm}$ $\times l=100 \mathrm{~mm}$. The total gamma efficiency of this setup was estimated to be $70 \%$ at $5 \mathrm{MeV}$. In our setup a Ge detector was placed at the collection point in order to monitor the composition of the sources. Our original goal was to measure the decay of ${ }^{102,104,105} \mathrm{Tc}$ but some technical problems during our run made impossible to measure the decay of ${ }^{102} \mathrm{Tc}$, so in the following we will discuss the measurement of the $\beta$ decay of ${ }^{104,105} \mathrm{Tc}$ isotopes.

A problem in the TAGS measurements is how to get rid of the possible contaminants. The importance of this problem is determined by the nature of the technique itself. A TAGS is a high efficiency summing device with bad resolution compared with a high resolution setup (Ge detector). For that reason it is of paramount importance to have clean sources. Since the production of the ${ }^{104,105} \mathrm{Tc}$ isotopes was high enough, we closed the slits as much as possible in the mass separator in order to eliminate the effect of contaminants with masses $A \pm 1$ around the mass $A$ of interest. The tight collimation represented a loss of approximately $25 \%$ of the isotopes of interest. Then the problem of the isobaric contamination has to be addressed. In our particular cases it was possible to eliminate the isobaric contamination by means of selecting appropriate collection/measuring cycles.

\section{Analysis and conclusions}

For the analysis of the total absorption spectra we have to solve the so called "inverse problem" $\mathbf{d}=\mathbf{R}(B) \mathbf{f}$ where $\mathbf{d}$ represents the measured data, $\mathbf{R}$ is the response matrix of the detector, and $\mathbf{f}$ is the feeding distribution we wish to determine. The response function $\mathbf{R}$ depends on the detector and on the branching ratios of the levels in the daughter nucleus (B) and can only be calculated using Monte Carlo (MC) techniques. The analysis of the data presented here was carried out using the methods of analysis established by the Valencia group [5]. The response function was determined using the code GEANT4 [9]. The quality of the Monte Carlo simulations was tested comparing the result of the simulations with measured radioactive sources ${ }^{22,24} \mathrm{Na}$.

In the analysis of the ${ }^{104,105} \mathrm{Tc}$ decay we have used the Expectation Maximization Algorithm to solve the inverse 

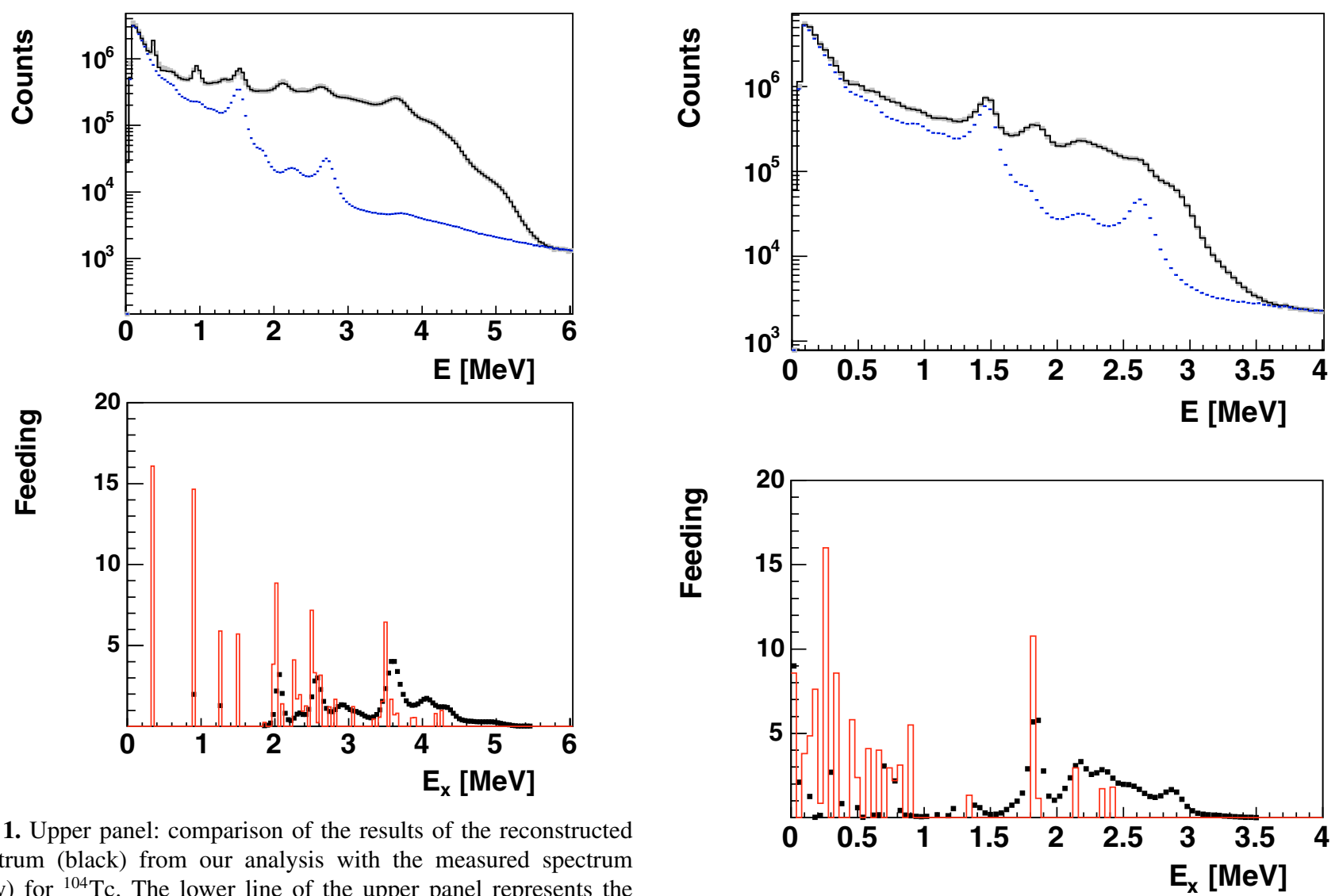
spectrum (black) from our analysis with the measured spectrum (gray) for ${ }^{104} \mathrm{Tc}$. The lower line of the upper panel represents the contribution of contaminants (background and pileup). On the lower panel the deduced feeding is given (dots), compared with the feeding previously known from high resolution measurements (discrete lines) [10].

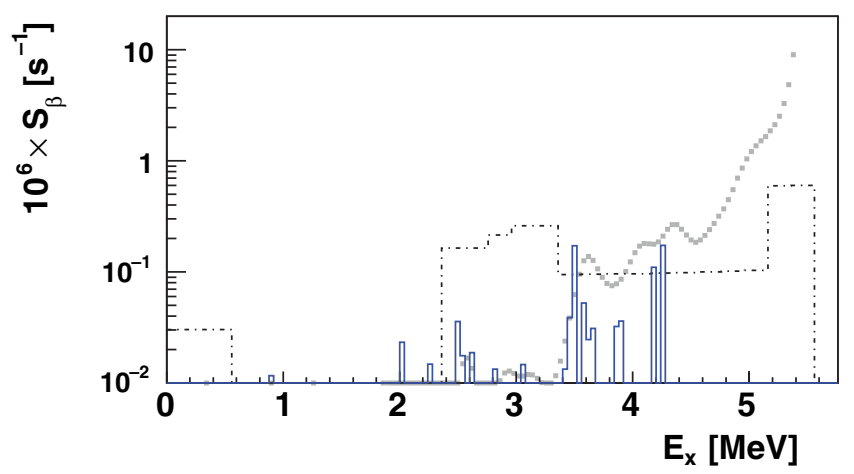

Fig. 2. Comparison of the deduced strength for ${ }^{104} \mathrm{Tc}$ (gray) with the strength predicted by Gross Theory (dotted line). The discrete lines represent the strength known from high resolution studies [10].

problem. As mentioned before the response matrix of the detector depends on the level scheme of the daughter nucleus we analyze. Several assumptions can be made for the level scheme. In the ${ }^{104} \mathrm{Tc}$ case we have used the known adopted level scheme [10] up to an excitation of $1556 \mathrm{keV}$ and from the $1840 \mathrm{keV}$ excitation up to the the Q value of 5516(6) $\mathrm{keV}$ [11] we have used the statistical nuclear model to generate an average branching ratio matrix. Once the level scheme and the branching ratio matrix are defined, $\mathbf{R}(B)$ is constructed from the individual gamma responses calculated in the $\mathrm{MC}$

Fig. 3. Upper panel: comparison of the results of the reconstructed spectrum (black) from our analysis with the measured spectrum (gray) for ${ }^{105} \mathrm{Tc}$. This result was obtained assuming a ground state feeding of $9 \%$ to the ground state of ${ }^{105} \mathrm{Ru}$. The lower line of the upper panel represents the contribution of contaminants (background and pileup). On the lower panel the deduced feeding from this analysis is given (dots), compared with the one previously known from high resolution measurements (discrete lines) [12].

simulations as explained in refs [5,6]. In the ${ }^{104} \mathrm{Tc}$ analysis presented here we have assumed negative parity for the levels at excitation $1502,1515 \mathrm{keV}$ in ${ }^{104} \mathrm{Ru}$ and only allowed Gamow-Teller transitions were considered. The spin-parity of the ground state of ${ }^{104} \mathrm{Tc}$ was assumed $3^{+}$. The results of our preliminary analysis for ${ }^{104} \mathrm{Tc}$ are presented in figures 1 and 2 .

In the ${ }^{105} \mathrm{Tc}$ case we have assumed a known level scheme up to the excitation $1325 \mathrm{keV}$ [12] and the statistical nuclear model was used to generate the average branching ratio matrix from the excitation of $1360 \mathrm{keV}$ up to the $\mathrm{Q}$ value of 3746(6) $\mathrm{keV}$ [11]. Compared to the ${ }^{104} \mathrm{Tc}$ case the ${ }^{105} \mathrm{Tc}$ case is more uncertain since the spin-parity assignment of the ground state of the parent is $\left(3 / 2^{-}\right)$and most of the low lying levels of ${ }^{105} \mathrm{Ru}$ fed in the decay according to [12] have positive parity. For that reason we have allowed feeding to all low lying levels in the analysis excluding only levels at excitation 229.5 and 301.7 that have $7 / 2^{+}$spin-parity assignment. Another uncertainty of this analysis is related to the fact that in our measurement we were not able to determine the ground state feeding and in the latest compilation [12] an upper limit to this feeding is given $(<9 \%)$. For that reason the results of two analysis as limiting cases are presented here in which the 


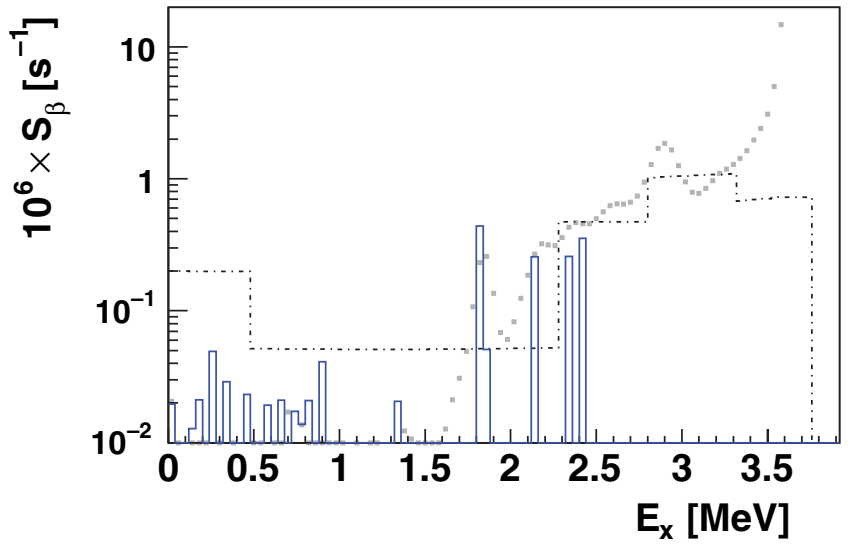

Fig. 4. Comparison of the deduced strength for ${ }^{105} \mathrm{Tc}$ (gray) with the strength predicted by Gross Theory (dotted line). This result was obtained assuming a ground state feeding of $9 \%$ to the ground state of ${ }^{105} \mathrm{Ru}$. The discrete lines represent the strength known from high resolution studies [12].

feeding to the ground state of ${ }^{105} \mathrm{Ru}$ is first set to $9 \%$, and in the second is set to $0 \%$. The results of our preliminary analysis for ${ }^{105} \mathrm{Tc}$ are shown in figures 3 and 4 for the first assumption of fixing the ground state feeding to $9 \%$.

In the upper panel of figures 1 and 3 the analyzed spectra (in gray) are compared with the spectra generated from the feeding pattern determined in our analysis (black) for ${ }^{104,105} \mathrm{Tc}$ respectively. In the lower panel the results for the deduced beta feeding are showed compared with the feeding previously known from high resolution measurements. It is clear from both Figures that a large amount of beta feeding is observed in our experiment at high excitation in the daughter which was not previously seen in high resolution experiments $[10,12]$.

In figures 2 and 4 the deduced strength from our analysis for the ${ }^{104,105} \mathrm{Tc}$ cases is presented. Also for comparison the strength predicted by Gross Theory and the strength deduced from high resolution measurements is shown. Again it is clear from both figures that a large amount of strength is observed in our experiment which was not previously seen in high resolution measurements. This pattern resembles to what is needed to explain the gamma-ray discrepancy and it is comparable with the predictions of the Gross Theory for the ${ }^{104,105}$ Tc cases.

Table 1. Comparison of mean gamma and beta energies included in the Jeff 3.1 database with the results of the preliminary analysis of our measurements for the decay of the ${ }^{104,105} \mathrm{Tc}$ isotopes. In the ${ }^{105} \mathrm{Tc}$ case two results are presented depending on the conditions set in the analysis. Case a) means that in the analysis we have assumed a feeding of $9 \%$ to the ground state of the daughter nucleus ${ }^{105} \mathrm{Ru}$. Case b) means that the feeding to the ground state in ${ }^{105} \mathrm{Ru}$ was fixed to zero.

\begin{tabular}{ccccc}
\hline Nuclide & $\begin{array}{c}\mathrm{E}_{\gamma} \\
\text { Jeff 3.1 }\end{array}$ & $\begin{array}{c}\mathrm{E}_{\gamma} \\
\text { TAGS }\end{array}$ & $\begin{array}{c}\mathrm{E}_{\beta} \\
\text { Jeff 3.1 }\end{array}$ & $\begin{array}{c}\mathrm{E}_{\beta} \\
\text { TAGS }\end{array}$ \\
\hline${ }^{104} \mathrm{Tc}$ & $1890(30)$ & $3263(65)$ & $1595(75)$ & $915(35)$ \\
${ }^{105} \mathrm{Tc}_{a}$ & $668(19)$ & $1736(70)$ & $1310(173)$ & $805(35)$ \\
${ }^{105} \mathrm{Tc}_{b}$ & $668(19)$ & $1893(70)$ & $1310(173)$ & $732(35)$ \\
\hline
\end{tabular}

The results of our preliminary analysis were used to calculate the mean gamma and beta energies released in the decay. These values are presented in table 1, compared to the values taken from the database Jeff 3.1. The errors quoted here should be considered conservative and include the influence of several experimental effects. The preliminary character of the present work is related to the realization of the second experiment in a much cleaner enviroment (using JYLTRAP). This data was measured only recently and we are in the process of sorting it. Ideally the final analysis for ${ }^{104,105} \mathrm{Tc}$ should be done using this data and the evaluation of the errors should also include the effect of the variation of the branching ratio matrix. We are presently working on this topic. It is clear from our present results that there is a large increase in the mean gamma energy relesed in the decay of ${ }^{104,105} \mathrm{Tc}$ compared with the high resolution results. When our mean gamma energies are incorporated in the ENDF decay data library [13], a large part of the discrepancy in the gamma component of the decay heat for ${ }^{239} \mathrm{Pu}$ is already resolved. The effect is less dramatic for ${ }^{235} \mathrm{U}$ because of the difference of the fission yields.

This work was supported by the following projects: Spanish MEC FPA 2002-04181-004-03 and FPA 2005-03993; OTKA NK69035; the EC contract MERG-CT-2004-506849 and the EURONS project; A. A. recognizes partial support from the Hung. Bolyai fellowship and from the Spanish Ramón y Cajal fellowship.

\section{References}

1. T. Yoshida, T. Tachibana, F. Storrer, K. Oyamatsu, J. Katakura, J. Nucl. Sci. Technol. 36, 135 (1999).

2. A.L. Nichols, FJ/OH Summer School 2000, and references therein.

3. J.C. Hardy, L.C. Carraz, B. Jonson, P.G. Hansen, Phys. Lett. B 71, 307 (1977).

4. Z. Hu et al., Phys. Rev. C 60, 024315 (1999); Z. Hu et al., Phys. Rev. C 62, 064315 (2000); J. Agramunt et al., Proc. of the SGR97 on New Facet of Spin Giant Resonances in Nuclei, Tokyo, Japan, edited by H. Sakai, H. Okamura, T. Wakasa (World Scientific, Singapore, 1998), p. 150; A. Algora et al., Nucl. Phys. A 654, 727c (1999); E. Nacher et al., Phys. Rev. Lett. 92, 232501-1 (2004); E. Poirier et al., Phys. Rev. C 69, 034307 (2004).

5. D. Cano-Ott, Ph.D. thesis, University of Valencia, 2000, J.L. Tain, D. Cano-Ott, Nucl. Instrum. Meth. A 571, 719 (2007).

6. J.L. Tain (these proceedings).

7. K. Takahashi, M. Yamada, Progr. Theor. Phys. 41, 1470 (1969); S. Koyama, K. Takahashi, M. Yamada, Progr. Theor. Phys. 45, 663 (1970); K. Takahashi, Progr. Theor. Phys. 45, 1466 (1971).

8. T. Tachibana, M. Yamada, Y. Yoshida, Progr. Theor. Phys. 84, 641 (1990); H. Nakata, T. Tachibana, M. Yamada, Nucl. Phys. A 594, 27 (1995); T. Tachibana, M. Yamada, Proc. Int. Conf. on Exotic Nuclei and Atomic Masses, Arles (1995), p. 763.

9. The GEANT 4 program package; S. Agostinelli et al., Nucl. Instrum. Meth. A 506, 250 (2003).

10. J. Blachot, Nucl. Data Sheets 64, 1 (1991).

11. T. Eronen, I. Moore (private communication).

12. D. de Frenne, E. Jacobs, Nucl. Data Sheets 105, 775 (2005).

13. A. Sonzogni (private communication). 